\title{
Color Atlas of Pathophysiology Authors: Stefan Silbernagl and Florian Lang (2016) 2016448 pp, 195 illustrations, Paperback/softback ISBN: 9783131165534 Thieme Publishers New York/Stuttgart
}

\author{
Anna-Karina Maier $^{1} \cdot$ Olaf Strauss $^{1}$
}

Received: 16 August 2016 / Accepted: 24 August 2016/Published online: 3 September 2016

(C) Springer-Verlag Berlin Heidelberg 2016

The Color Atlas of Physiology and the Color Alas of Pathophysiology both have a long lasting tradition among textbooks and have gained a well-known high reputation. This has developed from the condensed clear information, knowledge presentation of logical principles, and brilliant illustrations. This concept is guaranteed by the two authors, Stefan Silbernagl and Florian Lang, both outstanding physiologists and brilliant teachers.

The $3^{\text {rd }}$ edition of the Color Atlas of Pathophysiology is a convincing continuation of this concept of success. It uses the same principle with a text description on the left page and the illustration on the right page. The area of pathophysiology is divided into ten different fields, all of them in different color codes. In this way the reader can quickly access the information required. The illustrations have been improved in this edition. Although they are focused and concise, the illustrations still transfer the complicated contexts and interactions that represent the chain of events leading to disease. This succeeds even in representations of mechanisms that range from the complete body system to molecules. In this way the illustrations are helpful, since they are easy to remember. A great help is that the book starts with basic mechanisms. These basic mechanisms can be applied as modules that organize the many connections in physiologic organization. In addition to this, every chapter follows the same principle. They commence with a description of the basic physiology and use further this knowledge to explain pathophy siology. This concept of logic principles is a great help for the clinician to understand the many diseases and treatments by their causation and not by their variability in appearance. However, the knowledge of the Color Atlas of Physiology might be required for the beginner because the introductory description of basic physiology might be too compact. Also, the beginner may at first be confused by the large number of abbreviations.

The descriptions of eye diseases are embedded in the chapter on neurology - neuromuscular and sensory systems. It is divided into three parts: a.) diseases of the anterior chamber including refractive errors, cataract, and glaucoma; b.) diseases of the retina including Retinitis pigmentosa, inherited macular dystrophies, diabetic retinopathy, central artery occlusion and color blindness; c.) diseases of the visual pathway and the processing in the visual cortex. With this collection most important causes for visual impairment or blindness are covered. However, age-dependent macular degeneration is lacking in this list. Again, the authors succeeded in delivering basic principles such as the differences between diseases in the inner and the outer retina.

All in all, the new edition establishes again the highest standard. The didactic concept is again refined and brilliant. The chapters about eye diseases profit from the strong concept of summarizing the physiological and pathophysiological connections by logic concepts that appear simple, but do not reduce complexity. The book should be fully recommended.

Olaf Strauss

olaf.strauss@charite.de

Virchowklinikum (CVK), Augustenburger Platz 1,

13353 Berlin, Germany 